\title{
BMJ Open The Choice and Partnership Approach to community mental health and addictions services: a realist-informed scoping review protocol
}

\author{
Leslie Anne Campbell, ${ }^{1,2,3}$ Sharon E Clark, ${ }^{4}$ Caitlyn Ayn (D),${ }^{1}$ Jill Chorney, ${ }^{4,5}$ \\ Debbie Emberly, ${ }^{4}$ Julie MacDonald, ${ }^{6}$ Adrian MacKenzie, ${ }^{1,7}$ Daniel Marsh, ${ }^{8}$ \\ Kylie Peacock, ${ }^{1}$ Lori Wozney ${ }^{9}$
}

To cite: Campbell LA, Clark SE, Ayn C, et al. The Choice and Partnership Approach to community mental health and addictions services: a realist-informed scoping review protocol. BMJ Open 2019;9:e033247. doi:10.1136/ bmjopen-2019-033247

- Prepublication history and additional material for this paper are available online. To view these files, please visit the journal online (http://dx.doi. org/10.1136/bmjopen-2019033247).

Received 26 July 2019 Revised 25 0ctober 2019 Accepted 18 November 2019

Check for updates

(C) Author(s) (or their employer(s)) 2019. Re-use permitted under CC BY-NC. No commercial re-use. See rights and permissions. Published by BMJ.

For numbered affiliations see end of article.

Correspondence to Dr Leslie Anne Campbell; leslie.anne.campbell@dal.ca

\section{ABSTRACT}

Introduction Early identification and appropriate treatment of child and adolescent mental health disorders can often be hampered by patchwork services with poorly planned or unclear pathways. The Choice and Partnership Approach (CAPA) is an evidence-based transformational model of community (community-based or outpatient) mental health and addictions services for children and adolescents that aims to better match services to needs and to improve timely access to care. CAPA has been variably implemented across jurisdictions but has not been comprehensively evaluated for its impact on system and client outcomes. Our research question is, 'To what degree does CAPA work, for whom and under what circumstances?'. The purpose of this review is twofold: (1) to gain an understanding of the extent and outcomes of the implementation of CAPA in community mental health and addictions services; and (2) to identify the role of context as it influences the implementation of CAPA and resulting client and system outcomes.

Methods and analysis We will conduct a realistinformed scoping review of the literature related to CAPA in either child and adolescent or adult community mental health and addictions services. Relevant studies, reports and documentation will be identified by searching the following online databases: MEDLINE, Embase, CINAHL, PsycINF0, Academic Search Premier, ERIC, Web of Science, Cochrane, Dissertations Abstracts, NCBI Bookshelf, PubMed Central and the Canadian Health Research Collection. The search strategy was developed by a health sciences library scientist and informed by a multidisciplinary team comprising methodological and content knowledge experts. The search will gather evidence from multiple online databases of peer-reviewed literature and grey literature repositories. All articles will be independently assessed for inclusion by pairs of reviewers. The key themes derived from a thematic analysis of extracted data will be presented in a narrative overview. Ethics and dissemination Research ethics review is not required for this scoping review. The results will be disseminated through meetings with stakeholders (including clients and families, clinicians and decisionmakers), conference presentations and peer-reviewed publication. The results of this review will inform an overarching programme of research, policy and quality
Strengths and limitations of this study

- This review will summarise the extent and outcomes of the implementation of Choice and Partnership Approach (CAPA) in community mental health and addictions services.

- A realist perspective will allow for the identification of context-specific factors that influence the implementation of CAPA.

- The protocol employs a rigorous study design using an established scoping review methodology informed by a realist perspective and is overseen by a multidisciplinary team with methodological and content expertise.

- Broad inclusion criteria allow the identification of available evidence and any existing gaps.

- Included studies will not be appraised for quality as this review aims to include all available evidence.

indicator development to ultimately improve mental health and addictions care and subsequent mental health outcomes for children and adolescents.

\section{INTRODUCTION}

Mental health and addictions disorders are the most common sources of morbidity among children and youth in developed countries, affecting as many as one in five by the age of 15 years. ${ }^{1-5}$ In England, one in eight young people aged 5 to 19 years had one or more mental illnesses when assessed. ${ }^{6}$ Similarly, a survey of Australian youth showed that mental health disorders affected nearly one in seven 4 to 17 -year-olds within the previous 12 months. ${ }^{4}$ In New Zealand, scores from the Strengths and Difficulties Questionnaire were 'concerning' for approximately $8 \%$ of children aged 3 to $14 .^{7}$ The burden of mental illness among children is projected to increase in upcoming years; for example, the number of Canadian children living with 
a mental illness has been projected to increase by $20 \%$ by 2041. ${ }^{8}$

It has been well established that mental illness in childhood contributes to the disruption of educational and occupational attainment and to an increased risk of mental illness in adulthood. ${ }^{9}{ }^{10}$ Early identification and appropriate treatment are key both for optimising the mental health and development of youth during their childhood and adolescence and for improving long-term outcomes through adulthood. ${ }^{11}$ However, mental health services and supports are often patchwork in nature with poorly planned or unclear pathways, resulting in difficulties in navigation, mismatches between need and appropriate level of service and delays in access. ${ }^{12}$ Agencies providing child and adolescent mental health services in many developed countries report challenges in meeting demand that result in substantial wait times. ${ }^{13}$ Child and adolescent mental health and addictions services are increasingly under pressure to deliver timely care, often without adequate resources.

In Canada, fewer than one third of agencies report the ability to meet the Canadian Psychiatric Association's wait time benchmarks of 24 hours to emergency care, 2 weeks to urgent care and 1 month to scheduled care. ${ }^{13}$ Approximately $75 \%$ of Canadian children and youth do not receive specialised mental health services when they need them. ${ }^{1314}$ Similar concerns with long wait times have also been reported in other countries, such as the USA, the UK and elsewhere. ${ }^{15} 16$ Those children and youth that do receive care often receive treatments and interventions that are not based on evidence of efficacy or effectiveness. ${ }^{17}$ The Canadian Senate Report on the Status of Mental Health in Canada, entitled 'Out of the Shadows at Last' stated that without effective knowledge translation within mental health services, 'ineffective or even harmful treatments may continue, while effective, evidence-based treatments may not be adopted by policymakers and mental health service providers'. ${ }^{12}$ Although there are many successes in finding new evidence-based practices for mental illness and addictions treatment, the dissemination and implementation of these successes into clinical practice remains a major challenge. ${ }^{14}$

A review of child and adolescent mental health services in the UK identified the creation of accessible, childcentred and family-centred care that balance demands for services with their capacity to deliver care as areas for improvement. ${ }^{18}$ The Choice and Partnership Approach (CAPA) was developed to address these key issues. CAPA is a continuous improvement service model based on shared decision-making that is informed through outcome measurement to enhance the effectiveness of care and manage demand for services. ${ }^{19}$ CAPA incorporates several innovative features that differentiate it from traditional models of mental healthcare in that it is client and caregiver led, needs-based, accessible and outcome-focused. ${ }^{19} 20$ The philosophy underlying CAPA reflects a shift in clinician stance from 'expert with power' to 'facilitator or partner with expertise'.
The system incorporates elements of Lean processes, including quality measurement and clear, efficient pathways through the services that aim to avoid unnecessary waits. ${ }^{21}$ Eleven key components (Leadership, Language, Handle Demand, Choice Framework, Full Booking to Partnership, Selecting Clinician, Core and Specific Partnership Work, Job Plans, Goal Setting, Peer Group Discussion and Team Away Days) reflect the philosophical stance of family-centred care and the importance of the Lean process. $^{22}$

Efforts are under way in many developed countries to transform child and adolescent mental health and addictions services through increased funding to support new initiatives and expand resources. ${ }^{23-25}$ In some locations, CAPA has been selected to guide the transformation of child and adolescent mental health and addictions services with the intention of reducing wait times to services and improving timely access to evidence-based care. The CAPA model has been implemented in many community (community-based or outpatient) mental health and addictions programme within the UK, Australia, New Zealand, Norway, Belgium and Canada. ${ }^{22}$ Despite being an evidence-informed model, CAPA has not been comprehensively evaluated for fidelity of implementation and impact on system and client outcomes. For these reasons, our team is undertaking a realist evaluation of the implementation of CAPA within our region. Our overarching research question is, 'To what degree does CAPA work, for whom and under what circumstances?'.

To help inform our evaluation, as well as contribute to the literature, we will conduct a scoping review of the evidence. Initial keyword searching revealed minimal peer-reviewed evidence and some grey literature describing the use of CAPA and reporting some client and system outcomes, such as satisfaction with services and wait times. ${ }^{2026}$ Given the emerging and heterogenous nature of the literature regarding CAPA implementation, scoping methodology was deemed most appropriate to inform our evaluation, as it maps key concepts, provides an overview of the breadth and depth in identification of sources and does not restrict eligibility based on traditional hierarchies of evidence. ${ }^{27} 28$

We have adopted a realist-informed review approach as context-sensitive findings are of particular interest for shaping initial programme theories for our realist evaluation of the implementation of the CAPA model in a Canadian province. The purpose of this review is therefore twofold: (1) to gain an understanding of the extent and outcomes of the implementation of CAPA in community mental health and addictions services; and (2) to identify how context influences the implementation of CAPA and resulting client and system outcomes. Context may be described at the health system (eg, policies supportive of measurement-based care), clinic (eg, information technology systems that facilitate job planning or outcome measurement), clinician (eg, experiences, beliefs and attitudes of clinicians) or client (eg, mental healthcare needs or expectations) levels. 


\section{METHODS AND ANALYSIS}

As part of our realist-informed scoping review methodology, we are drawing on an overarching framework for our review using the Consolidated Framework for Implementation Research (CFIR) to provide structure for describing and understanding context and the implementation of CAPA and for framing our initial programme theories. ${ }^{272930}$ The CFIR comprises five major domains: intervention characteristics, outer setting, inner setting, characteristics of the individuals involved and the process of implementation. The framework was selected as it consolidates various implementation theories, ensuring thorough capture of relevant constructs and promotes consistent terminology and definitions. ${ }^{30}$

To ensure this synthesis would be a novel contribution to the literature, an initial scan of review literature was undertaken with explicit searching using the term 'Choice and Partnership Approach'. This search was run in Database of Promoting Health Effectiveness Reviews (DoPHER; EPPI), Cochrane, Epistemonikos, Joanna Briggs Institute Database of Systematic Reviews and Implementation Reports (JBISRIR; Wolters Kluwer), PROSPERO (University of York), Campbell Collaboration, HealthEvidence.org (McMaster University), PubMed (NCBI) and CINAHL (EBSCO). No relevant results were retrieved from these initial searches.

We adhered to the Preferred Reporting Items for Systematic Reviews and Meta-Analyses (PRISMA) for scoping reviews as well as the relevant Realist And Meta-narrative Evidence Syntheses: Evolving Standards (RAMESES) in developing our protocol. ${ }^{31}{ }^{32}$ This scoping review follows the framework proposed originally by Arksey and O'Malley and revised by Levac and colleagues, and is informed by the realist review methods developed by Pawson and colleagues. ${ }^{27-29}$

Briefly, the stages are:

1. Identifying the research question;

2. Identifying relevant studies;

3. Selecting studies;

4. Charting the data;

5. Collating, summarising and reporting results;

6. Consulting with stakeholders.

\section{Stage 1: identifying the research question}

Our overarching research question is, 'To what degree does CAPA work, for whom and under what circumstances?'. Our realist-informed scoping review will examine the literature related to the CAPA in community mental health and addictions services.

While CAPA was developed in child and adolescent community mental health services, we will also include literature from adult mental health services in our review to comprehensively map the availability of evidence. As our review will also serve to support a realist evaluation of the implementation of CAPA in child and adolescent mental health and addictions services in a Canadian province (Nova Scotia) in which youth are treated up to and including age 18 years, studies serving primarily adult populations may include our age group of interest. CAPA is also being implemented by adult services, and as such the results will inform those efforts.

We will not generalise the question to include the components of CAPA (such as shared decision-making or goal-based outcome measurement) as these are clinical practices in their own right and individually do not capture the comprehensive nature of CAPA as a model of care.

\section{Stage 2: identifying relevant studies}

Levac and colleagues recommend combining a broad research question with a clearly defined scope of inquiry. ${ }^{28}$ Relevant studies, reports and documentation will be identified by searching the following online databases: MEDLINE, Embase, CINAHL, PsycINFO, Academic Search Premier, ERIC, Web of Science, Cochrane, Dissertations Abstracts, NCBI Bookshelf, PubMed Central and the Canadian Health Research Collection. A wide range of databases was selected to include the breadth of literature from healthcare, psychology, social work and social sciences. Since this review is specific to CAPA, evidence published from 1 January, 2005, to the date of the search will be included, as foundational elements of CAPA were published during this period. ${ }^{33}$ No restrictions due to language or country of origin will be applied. Materials will be translated to English as needed.

The peer-reviewed database search strategy was co-developed by the study's principal and co-investigators and library scientists. We first conducted a preliminary search to gain familiarity with CAPA-related literature and terminology. Following this, a search query was constructed and reviewed by the principal investigator. The resulting search query used in MEDLINE (Ovid) and CINAHL (EBSCO) is shown in table 1. Given the iterative nature of scoping reviews, the search strategy may be further refined or expanded as new terminology or data sources are identified. The database searches will be completed by a library scientist with supervision from the study principal and co-investigators. The searches will be saved and monitored regularly for any newly published material throughout the review process. The reference lists of all included studies will also be hand searched.

Grey literature searching will be undertaken to ensure any unpublished, novel and non-traditional data sources are included. The peer-reviewed database search strategy will be translated through consultations with a library

Table 1 Sample peer-reviewed database search queries

MEDLINE ('choice and partnership* OR

(choice and partnership*' adj2 (approach* OR model OR models

OR program OR programs OR programme

OR programmes))).ti,ab,kw,kf.

CINAHL ('choice and partnership*' N2 (approach or approaches or model or models or program\$)) in ti,ab,subject 
scientist and run in commonly-used grey literature repositories, such as those curated by the Canadian Agency for Drugs and Technologies in Health $(\mathrm{CADTH}) .{ }^{34}$ In addition, terms including 'Choice and Partnership Approach' and 'CAPA' will be run in search engines. General searches will be conducted to retrieve web content and advanced searching will be used to identify content with commonlyused file types. Results from the first 10 pages (sorted by relevance) will be documented. Any potentially-relevant webpages will be searched in greater depth. As grey literature searching is highly iterative, further approaches may be identified throughout the review process. ${ }^{35}$

Scoping reviews both benefit from and often struggle with achieving a balance of breadth and feasibility. ${ }^{27} 28$ Our study team includes members with both methodological and content expertise to inform decisions regarding breadth and feasibility. Any potential limitations arising from these decisions or revisions of the search strategy will be acknowledged in our review.

\section{Stage 3: selecting studies}

In keeping with scoping review methodology, our interdisciplinary team will employ a rigorous approach to study selection using a transparent and replicable process. ${ }^{27}{ }^{28}{ }^{36} \mathrm{We}$ are not restricting our approach to a strict paradigm following a hierarchical consideration of evidence. We will document each step of the selection process, including decisions made regarding inclusion and exclusion of studies.

The following inclusion criteria will be used to guide decision-making:

1. Study or report focuses on the CAPA model, including its implementation, outcomes or a discussion of contextual factors that may impact implementation.

2. Outcomes are not restricted, and may include clinical, programme or system outcomes.

3. Population of interest may include child and adolescent or adult populations in community mental health and addictions service settings.

4. Context is not limited and may include any site or service that has implemented CAPA or is in the process of transitioning to CAPA at the level of system, service, clinic, clinician or client.

5. May include any methodology or type of publication.

The study selection process itself involves two steps: title/ abstract screening, followed by full-text review. References for all documents retrieved through the search will be managed using the Covidence platform. Two investigators (LAC and SC) will independently screen titles/abstracts and will respond 'yes', 'no' or 'maybe' when prompted by the platform. Any documents whose eligibility cannot be determined from the title/abstract screening will be moved to full-text review for more detailed assessment. Following title/abstract screening, the reviewers will read the full-text of the remaining articles and assign them as 'include' or 'exclude'. The reviewers will meet at the beginning, midpoint and end of the process to discuss challenges and resolve any ambiguity with the inclusion criteria. Discrepant or uncertain assignments will be assigned through consensus-based discussion between the reviewers. Inclusion of a third investigator (JM) or full study team discussions may also be utilised to resolve discrepancies, if necessary.

\section{Stage 4: charting the data}

We will extract data that correspond to key categories from each of the included studies. A draft codebook (data extraction form) has been developed to reflect the domains of the CFIR Framework, the team's content knowledge and relevant publications. Data elements to be extracted include document identification, objectives, methods, context, implementation, outcomes and takeaways (see online supplementary file 1 for the draft categories included in the codebook). Following the recommendation of Levac and colleagues, we will conduct the data charting in an iterative manner, updating the codebook as appropriate based on our findings. ${ }^{28}$ Two team members (LAC and SC) will independently extract the data from the first three studies using the codebook and will review to verify consistency with the identified research question and purpose.

\section{Stage 5: collating, summarising and reporting the results}

The aim of our summary of results is not to aggregate findings from the included studies, but rather to present a narrative synthesis of the material retrieved and reviewed, and to identify gaps in knowledge. ${ }^{27}$ We will use our findings to develop initial programme theories underlying the assumptions about how CAPA is meant to work and what impacts it is expected to have, which will inform our realist evaluation. ${ }^{29}$

We will begin with a simple descriptive analysis of the extracted data using basic frequencies and ranges to provide a numerical summary of the findings. Contextual and process-oriented data will be analysed using thematic analysis to identify key emerging themes based on the CFIR framework. ${ }^{30}$ The resulting themes will be reviewed by content expert team members to ensure validity and credibility. Similarities and differences between studies and gaps in the literature will be reported.

The results from empirical qualitative, quantitative and mixed methods studies as well as grey literature reports will be classified into the five major domains and associated constructs outlined in the modified CFIR framework (as presented in table 2) and informed by our CAPA logic model. Finally, results will be summarised in a narrative analysis.

\section{Stage 6: consulting with stakeholders}

While considered an optional stage in conducting a scoping review by Arksey and O'Malley, we believe consultation is an essential component to our review, adding rigour and depth to the results. ${ }^{27}$ Engaged stakeholders include clinicians, administrators, clients, families, researchers and policymakers. The purpose of our consultation is to identify key outcomes of interest, validate the findings, contextualise results and inform the 
Table 2 Summary framework (adapted from the Consolidated Framework for Implementation Research)

\begin{tabular}{|c|c|}
\hline Domain & Relevant constructs \\
\hline $\begin{array}{l}\text { Intervention } \\
\text { characteristics }\end{array}$ & $\begin{array}{l}\text { Evidence strength (quality will not be } \\
\text { assessed) } \\
\text { Relative advantage } \\
\text { Adaptability } \\
\text { Trialability } \\
\text { Complexity } \\
\text { Design quality/packaging } \\
\text { Cost }\end{array}$ \\
\hline Outer setting & $\begin{array}{l}\text { Client needs and resources } \\
\text { Cosmopolitanism (networking to other } \\
\text { external organisations) } \\
\text { Peer pressure } \\
\text { External policies and incentives }\end{array}$ \\
\hline
\end{tabular}

\begin{tabular}{|c|c|}
\hline Inner setting & $\begin{array}{l}\text { Structural characteristics (social } \\
\text { architecture, age, maturity and size of } \\
\text { organisation) } \\
\text { Networks and communications } \\
\text { Culture } \\
\text { Implementation climate }\end{array}$ \\
\hline $\begin{array}{l}\text { Characteristics of } \\
\text { individuals }\end{array}$ & $\begin{array}{l}\text { Knowledge and beliefs about CAPA } \\
\text { Self-efficacy } \\
\text { Individual stage of change } \\
\text { Individual identification with } \\
\text { organisation } \\
\text { Other personal attributes }\end{array}$ \\
\hline $\begin{array}{l}\text { Process of } \\
\text { implementation }\end{array}$ & $\begin{array}{l}\text { Planning } \\
\text { Engaging } \\
\text { Executing } \\
\text { Reflecting and evaluating }\end{array}$ \\
\hline
\end{tabular}

CAPA, Choice and Partnership Approach.

development of knowledge translation tools. Consultation may also reveal sources of evidence not found through traditional database searching. Based on our contacts and initial search, we anticipate consulting with 12 to 20 individuals to ensure representation of the various stakeholders and to achieve saturation. We will facilitate consultation through interviews and/or focus groups through our collaborations with local, national and international stakeholders.

\section{PATIENT AND PUBLIC INVOLVEMENT STATEMENT}

Clients (patients) and caregivers are integral to the CAPA model and are key partners in our research. We have an overarching strategy for client and caregiver engagement that supports clients and caregivers and provides multiple opportunities to partner with the research team in a variety of formats (eg, healthyyoungminds.ca). Throughout our evaluation, clients and caregivers will inform participant recruitment strategies, identify outcomes of interest, vet knowledge translation materials and co-develop research directions stemming from the review.

\section{ETHICS AND DISSEMINATION}

As a review of existing literature this research does not require ethics approval.

The results will be disseminated through meetings with stakeholders (including clients and families, clinicians and decision-makers), conference presentations and peerreviewed publication. Our dissemination plan includes using our findings to inform a larger programme of research, and policy and quality indicator development, to support evidence-based mental health and addictions care and ultimately improve the mental health outcomes of children and adolescents. Our integrated knowledge translation strategy is particularly important for developing approaches to engage clients, families, clinicians and decision-makers in the implementation and ongoing evaluation of mental health and addictions services. Activities will contextualise our results and provide insights not available from data alone.

This scoping review will be useful for services looking to transition to the CAPA model by identifying potential challenges to implementation and sustainability. It will also be used to generate initial programme theories that may explain how CAPA works in practice as part of our realist evaluation of CAPA in Nova Scotia, Canada.

\section{Author affiliations}

${ }^{1}$ Community Health \& Epidemiology, Dalhousie University, Halifax, Nova Scotia, Canada

${ }^{2}$ School of Nursing, Dalhousie University, Halifax, Nova Scotia, Canada

${ }^{3}$ Psychiatry, IWK Health Centre, Halifax, Nova Scotia, Canada

${ }^{4}$ Mental Health and Addictions, IWK Health Centre, Halifax, Nova Scotia, Canada

${ }^{5}$ Anesthesia, Pain Management \& Perioperative Medicine, and Psychology \&

Neuroscience, Dalhousie University, Halifax, Nova Scotia, Canada

${ }^{6}$ Mental Health and Addictions, Nova Scotia Health Authority, Sydney, Nova Scotia, Canada

${ }^{7}$ Health Workforce Planning and Regulatory Affairs Branch, Nova Scotia Department of Health and Wellness, Halifax, Nova Scotia, Canada

${ }^{8}$ Administration, Nova Scotia Health Authority, Halifax, Nova Scotia, Canada

${ }^{9}$ Mental Health and Addictions, Nova Scotia Health Authority, Dartmouth, Nova Scotia, Canada

Acknowledgements The authors are grateful for the support provided by the Maritime SPOR SUPPORT Unit (https://www.spor-maritime-srap.ca/) in the form of consultation with the Evidence Synthesis Coordinator for the development and conduct of the search strategy. We are also grateful to Robin Parker, MLIS, for her suggestions for refining the search. We thank Justin Jagosh, PhD, for his suggestions for incorporating the realist evaluation component.

Contributors $L A C$ is the principal investigator and led all aspects of protocol development. LAC, SC, JM and DM determined the review objectives and eligibility criteria. LAC and SC refined the review approach. $L A C, L W, D E, C A$ and KP developed the data extraction template. LAC and KP developed the client and caregiver engagement strategy. LAC and CA conducted the preliminary search of review literature. $L A C$ reviewed and refined the initial peer-reviewed database search strategy drafted by research librarian staff. LAC and CA developed the grey literature search approach. SC, JM, LW, JC and AM provided critical commentary on manuscript content. All authors approved the final version of the manuscript.

Funding This work was supported by Research Nova Scotia (formerly the Nova Scotia Health Research Foundation) grant number 1564 in the form of an Establishment Grant awarded to LA Campbell (Principal Investigator).

Competing interests None declared.

Patient consent for publication Not required.

Provenance and peer review Not commissioned; externally peer reviewed. 
Data availability statement № data are available.

Open access This is an open access article distributed in accordance with the Creative Commons Attribution Non Commercial (CC BY-NC 4.0) license, which permits others to distribute, remix, adapt, build upon this work non-commercially, and license their derivative works on different terms, provided the original work is properly cited, appropriate credit is given, any changes made indicated, and the use is non-commercial. See: http://creativecommons.org/licenses/by-nc/4.0/.

ORCID iD

Caitlyn Ayn http://orcid.org/0000-0001-5114-2904

\section{REFERENCES}

1 Offord DR, Boyle MH, Szatmari P, et al. Ontario child health study. II. six-month prevalence of disorder and rates of service utilization. Arch Gen Psychiatry 1987;44:832-6.

2 Waddell C, Offord DR, Shepherd CA, et al. Child psychiatric epidemiology and Canadian public policy-making: the state of the science and the art of the possible. Can J Psychiatry 2002;47:825-32.

3 Waddell C, McEwan K, Shepherd CA, et al. A public health strategy to improve the mental health of Canadian children. Can J Psychiatry 2005:50:226-33.

4 Lawrence D, Johnson S, Hafekost J. The Mental Health of Children and Adolescents : Report on the second Australian Child and Adolescent Survey of Mental Health and Wellbeing. Available: https:// research.acer.edu.au/well_being/1/ [Accessed 16 Jun 2019].

5 Sadler K, Vizard T, Ford T. Mental health of children and young people in England, 2017; 2018.

6 NHS Digital. Mental health of children and young people in England, 2017. Available: https://digital.nhs.uk/data-and-information/ publications/statistical/mental-health-of-children-and-young-peoplein-england/2017/2017 [Accessed 16 Jun 2019].

7 Ministry of Health NZ. Social, emotional and behavioural difficulties in New Zealand children: new Zealand health survey. Available: https:// www.health.govt.nz/publication/social-emotional-and-behaviouraldifficulties-new-zealand-children-new-zealand-health-survey [Accessed 16 Jun 2019].

8 Smetanin P, Stiff D, Briante C, et al. The life and economic impact of major mental illnesses in Canada: 2011 to 2041. Toronto: RiskAnalytica, on behalf of the Mental Health Commission of Canada, 2011

9 Kessler RC, Foster CL, Saunders WB, et al. Social consequences of psychiatric disorders, I: educational attainment. Am J Psychiatry 1995;152:1026-32.

10 Kim-Cohen J, Caspi A, Moffitt TE, et al. Prior juvenile diagnoses in adults with mental disorder: developmental follow-back of a prospective-longitudinal cohort. Arch Gen Psychiatry 2003;60:709-17.

11 Das JK, Salam RA, Lassi ZS, et al. Interventions for adolescent mental health: an overview of systematic reviews. J Adolesc Health 2016;59:S49-60.

12 Kirby MJ, Keon WJ Out of the shadows at last: highlights and recommendations final report of the standing Senate Committee on social Affairs, science and technology; 2006.

13 Kowalewski K, McLennan JD, McGrath PJ. A preliminary investigation of wait times for child and adolescent mental health services in Canada. $J$ Can Acad Child Adolesc Psychiatry 2011;20:112.

14 Ringel JS, Sturm R. National estimates of mental health utilization and expenditures for children in 1998. J Behav Health Serv Res 2001;28:319-33.
15 Steinman KJ, Shoben AB, Dembe AE, et al. How long do adolescents wait for psychiatry appointments? Community Ment Health J 2015;51:782-9.

16 Smith J, Kyle RG, Daniel B, et al. Patterns of referral and waiting times for specialist child and adolescent mental health services. Child Adolesc Ment Health 2018;23:41-9.

17 Hoagwood K, Olin SS. The NIMH blueprint for change report: research priorities in child and adolescent mental health. J Am Acad Child Adolesc Psychiatry 2002;41:760-7.

18 Davidson J. Children and young people in mind: the final report of the National CAMHS review. London: Department of Health, 2008.

19 York A, Kingsbury S. The choice and partnership approach: a transformational service model. UK: CAPA Publications, 2013.

20 Robotham D. Evaluation of the choice and partnership approach (CAPA) in child and adolescent mental health services in England; 2009.

21 Mazzocato P, Savage C, Brommels M, et al. Lean thinking in healthcare: a realist review of the literature. BMJ Qual Saf 2010;19:376-82.

22 York A, Kingsbury S. The choice and partnership approach: a guide to CAPA. CAMHS Network, 2009.

23 Department of Health and Wellness. Business plan 2018-19, 2018. Available: https://novascotia.ca/government/accountability/ 2018-2019/2018-2019-business-plan-Department-of-Health- andWellness.pdf

24 lyer SN, Boksa P, Lal S, et al. Transforming youth mental health: a Canadian perspective. Ir J Psychol Med 2015;32:51-60.

25 Malla A, lyer S, McGorry P, et al. From early intervention in psychosis to youth mental health reform: a review of the evolution and transformation of mental health services for young people. Soc Psychiatry Psychiatr Epidemiol 2016;51:319-26.

26 Clark S, Emberly D, Pajer K, et al. Improving access to child and adolescent mental health care: the choice and partnership approach. $J$ Can Acad Child Adolesc Psychiatry 2018;27:5-14.

27 Arksey H, O'Malley L. Scoping studies: towards a methodological framework. Int J Soc Res Methodol 2005;8:19-32.

28 Levac D, Colquhoun H, O'Brien KK. Scoping studies: advancing the methodology. Implement Sci 2010;5.

29 Pawson R, Greenhalgh T, Harvey G, et al. Realist review--a new method of systematic review designed for complex policy interventions. J Health Serv Res Policy 2005;10 Suppl 1:21-34.

30 Damschroder LJ, Aron DC, Keith RE, et al. Fostering implementation of health services research findings into practice: a consolidated framework for advancing implementation science. Implement Sci 2009;4.

31 Moher D, Shamseer L, Clarke M, et al. Preferred reporting items for systematic review and meta-analysis protocols (PRISMA-P) 2015 statement. Syst Rev 2015;4:1.

32 Wong G, Greenhalgh T, Westhorp G, et al. RAMESES publication standards: realist syntheses. BMC Med 2013;11:21.

33 York A, Kingsbury S. The 7 HELPFUL habits of effective CAMHS \& the choice and partnership approach: A workbook for CAMHS. London, 2005.

34 Canadian Agency for Drugs and Technologies in Health. Grey matters: a practical tool for searching health-related grey literature. Ottawa, ON; 2015.

35 Godin K, Stapleton J, Kirkpatrick SI, et al. Applying systematic review search methods to the grey literature: a case study examining guidelines for school-based breakfast programs in Canada. Syst Rev 2015:4:138.

36 Colquhoun HL, Levac D, O'Brien KK, et al. Scoping reviews: time for clarity in definition, methods, and reporting. $J$ Clin Epidemiol 2014;67:1291-4 